\title{
Impact of Stressful Life Events on Patients with Chronic Obstructive Pulmonary Disease
}

\author{
Tsung $\mathrm{Yu}^{\mathrm{a}, \mathrm{b}}$ Anja Frei ${ }^{\mathrm{a}}$ Gerben ter Riet ${ }^{\mathrm{c}}$ Milo A. Puhan ${ }^{\mathrm{a}}$ \\ ${ }^{a}$ Epidemiology, Biostatistics and Prevention Institute, University of Zurich, Zurich, Switzerland; \\ ${ }^{b}$ Department of Public Health, China Medical University and Big Data Center, China Medical University Hospital, \\ Taichung, Taiwan; ' Department of General Practice/Family Medicine, Academic Medical Center, University of \\ Amsterdam, Amsterdam, The Netherlands
}

\section{Keywords}

Stressful life events - Chronic obstructive pulmonary disease - ICE COLD ERIC cohort study · Depression · Anxiety $\cdot$ Physical activity

\begin{abstract}
Background: There is a general notion that stressful life events may cause mental and physical health problems. $\mathbf{O} \boldsymbol{b}$ jectives: We aimed to describe stressful life events reported by patients with chronic obstructive pulmonary disease (COPD) and to assess their impact on health outcomes and behaviors. Methods: Two hundred and sixty-six primary care patients who participated in the ICE COLD ERIC cohort study were asked to document any stressful life events in the past 3 years. We assessed the before-after (the event) changes for symptoms of depression and anxiety, health status, dyspnea-related quality of life, exacerbations, cigarette use, and physical activity. We used linear regression analysis to estimate the crude and adjusted magnitude of the before-after changes. Results: About 41\% (110/266) of patients reported the experience of any stressful life events and "death of relatives/important persons" was most common (31\%). After accounting for age, sex, living status, lung function, and anxiety/depression status at baseline, experiencing any stressful life events was associated with a 0.9-point increase on the
\end{abstract}

depression scale ( $95 \% \mathrm{Cl} 0.3$ to 1.4 ), a 0.8-point increase on the anxiety scale ( $95 \% \mathrm{Cl} 0.3$ to 1.3 ), and a 0.8-point decrease in the physical activity score $(95 \% \mathrm{Cl}-1.6$ to 0$)$. Conclusions: Experiencing stressful life events was associated with a small to moderate increase in symptoms of depression and anxiety in COPD, but no discernable effect was found for other physical outcomes. However, confirmation of these results in other COPD cohorts and identification of patients particularly vulnerable to stressful life events are needed.

두 2017 S. Karger AG, Base

\section{Introduction}

There is a general notion that psychological stress can cause mental and physical health problems $[1,2]$. In epidemiological studies, one way to quantify stress is by counting the occurrence of stressful life events. Since the landmark study by Holmes and Rahe [3] on the assessment of stressful life events, numerous studies have suggested their relations with conditions, including psychiatric disorders, cardiovascular diseases, cancers, and autoimmune diseases [4-8]. Two main pathways have been proposed that can link stressful life events (or stress in general) with health problems. Stressful life events can induce physiological adjustment, such as an increase in

\section{KARGER}

(c) 2017 S. Karger AG, Basel

E-Mail karger@karger.com

www.karger.com/res
Prof. Milo A. Puhan, MD, $\mathrm{PhD}$

Hirschengraben 84

CH-8001 Zurich (Switzerland)

E-Mail miloalan.puhan@uzh.ch 
blood pressure and stress hormones or a decrease in immune function. Stress might as well lead to behavioral changes, such as more use of tobacco or alcohol and less sleep or physical exercise $[1,2,9]$. Thus, stressful life events may increase the risk for many diseases.

In patients with chronic obstructive pulmonary disease (COPD), depression and anxiety have been widely investigated and were shown to increase patients' risk of exacerbations or death $[10,11]$. However, 1 potential cause of depression and anxiety, namely stressful life events, was rarely assessed in COPD cohorts. To the best of our knowledge, only in 1 cross-sectional study conducted in Singapore [12], the investigators documented that experiencing stressful life events was associated with more depressive symptoms in COPD patients than in their non-COPD counterparts. There is, at present, a lack of evidence from longitudinal cohort studies regarding the type of stressful life events experienced by COPD patients, the impact of stressful life events on patient outcomes, and the role that stressful life events play in COPD prognosis.

In this study, we explored the relationship between experiencing stressful life events and several outcomes/behaviors, including depression, anxiety, dyspnea, health status, exacerbations, cigarette use, and physical activity in patients enrolled in the International Collaborative Effort on Chronic Obstructive Lung Disease: Exacerbation Risk Index Cohorts (ICE COLD ERIC) study. Our aims were, firstly, to describe the stressful life events reported by COPD patients and, secondly, to quantify the impact of stressful life events on patient outcomes/behaviors.

\section{Methods}

\section{Population}

The study sample consisted of patients participating in the prospective ICE COLD ERIC cohort study, for which the design paper and the baseline characteristics of participants have been published previously $[13,14]$. Briefly, patients with COPD aged 40 years or older, in GOLD stage II-IV, and free of exacerbations for at least 4 weeks were recruited by their primary care physicians in the Netherlands or Switzerland from April 2008 to August 2009. The study exclusion criteria were being judged to have a life expectancy of no more than 12 months and being diagnosed with dementia, psychosis, or another psychiatric illness that may invalidate the assessment of patient-reported outcomes. We followed the patients for up to 5 years and assessed at half-year intervals the patient-reported outcomes on health status, symptoms of anxiety and depression, levels of physical activity, and health-related quality of life. At the 3.5-year follow-up visit, we administered an additional questionnaire on information on stressful life events that the patients had experienced in the past 3 years.
The ICE COLD ERIC study was approved by the medical ethics committees in Zurich (EK-1519) and Amsterdam (MEC-08-073). Each patient's written consent to the study was obtained by the investigators.

\section{Measures}

Patients completed the Hospital Anxiety and Depression Scale (HADS), the Chronic Respiratory Disease Questionnaire (CRQ), and the feeling thermometer at baseline and at half-year intervals [13]. The HADS is a valid and reliable instrument to measure depression and anxiety symptoms in patients with physical impairment, with domain scores ranging from 0 (no depression or anxiety at all) to 21 (very severe depression or anxiety) [15]. The CRQ is a valid and reliable instrument to measure disease-specific health-related quality of life in patients with COPD that includes domains of fatigue, dyspnea, emotional function, and mastery. Domain scores range from 0 (lowest level of quality of life) to 7 (highest) [16]. The feeling thermometer is a modified visual analogue scale with numerical values that range from 0 (worst imaginable health state) to 100 (best imaginable health state) to measure general health status in the past 7 days. It is part of the EuroQol 5 and has been validated for COPD patients $[17,18]$. Physical activity was assessed by the Longitudinal Ageing Study Amsterdam Physical Activity Questionnaire. As reported elsewhere [19], we generated a score ranging from 0 to 23 to indicate the level of physical activity at each assessment.

To assess stressful life events, at the 3.5-year follow-up, trained study nurses asked the patients to recall whether they had experienced in the past 3 years any life events that were so stressful that they seriously impacted their lives. Then they categorized these events into the following 9 categories: (1) death of relatives or important persons; (2) patient's own disease; (3) disease of relatives or important persons; (4) relational problems with relatives or important persons; (5) financial worries; (6) unemployment or retirement; (7) divorce, separation, or loss of friendship; (8) loss of pets, and (9) other. They also asked the patients to try to recall the date/ month/year of the occurrence of the events.

COPD exacerbations were assessed every 6 months based on patients' interviews and then centrally adjudicated by an expert panel who had access to patient charts and study case report forms, using the definition of exacerbations reported in the design paper [13].

\section{Statistical Analysis}

For each patient who had experienced at least 1 stressful life event, we computed the average of the HADS depression/anxiety scores, dyspnea-related quality of life scores, health status scores, physical activity scores, numbers of cigarettes smoked, and incidence rate of exacerbations (per person-year) that were measured before the occurrence of the first stressful life event ("before period") and after the first stressful life event ("after period"). In order to make comparisons, for each patient who had not experienced any stressful life events, we computed the average of scores/numbers or the incidence rate before visit 4 ("before period") and from visit 4 onwards ("after period"), because the median time of occurrence of stressful life events among those who had them was between visit 3 and 4 . We computed the difference between the 2 periods and used it as the dependent variable (before-after change) to represent the impact of stressful life events on health outcomes/behaviors.

We performed a linear regression analysis to assess if there was a difference between those who had any stressful life events and 
Table 1. Baseline characteristics of patients, stratified by patients' reporting of stressful life events

\begin{tabular}{|c|c|c|c|}
\hline \multirow[t]{2}{*}{ Characteristics } & \multirow[t]{2}{*}{ Total } & \multicolumn{2}{|c|}{ Any stressful life event reported } \\
\hline & & yes & no \\
\hline Patients, $n$ & 266 & 110 & 156 \\
\hline \multicolumn{4}{|l|}{ Sex, $n(\%)$} \\
\hline Male & $154(58)$ & $59(54)$ & $95(61)$ \\
\hline Female & $112(42)$ & $51(46)$ & $61(39)$ \\
\hline \multicolumn{4}{|l|}{ Nationality, $n(\%)$} \\
\hline Switzerland & $122(46)$ & $49(45)$ & $73(47)$ \\
\hline The Netherlands & $144(54)$ & $61(55)$ & $83(53)$ \\
\hline Mean age (SD), years & $66.0(9.4)$ & $64.7(9.6)$ & $66.9(9.2)$ \\
\hline Mean BMI (SD) & $26.4(5.0)$ & $26.6(5.2)$ & $26.3(4.9)$ \\
\hline \multicolumn{4}{|l|}{ Highest education, $n(\%)$} \\
\hline Primary school & $45(17)$ & $19(17)$ & $26(17)$ \\
\hline Secondary school & $147(55)$ & $60(55)$ & $87(56)$ \\
\hline Intermediate vocational & $44(17)$ & $18(16)$ & $26(17)$ \\
\hline Higher vocational/university & $26(10)$ & $12(11)$ & $14(9)$ \\
\hline \multicolumn{4}{|l|}{ Profession, $n(\%)$} \\
\hline Working or housewife & $75(28)$ & $40(36)$ & $35(22)$ \\
\hline Retired & $157(59)$ & $55(50)$ & $102(65)$ \\
\hline Disabled or unemployed & $34(13)$ & $15(14)$ & $19(12)$ \\
\hline \multicolumn{4}{|l|}{ Living situation, $n$ (\%) } \\
\hline Alone & $181(68)$ & $74(67)$ & $107(69)$ \\
\hline With partner or children & $83(31)$ & $36(33)$ & $47(30)$ \\
\hline Mean smoking pack-years (SD) & $44.3(27.7)$ & $44.2(25.8)$ & $44.4(29.1)$ \\
\hline Mean $\mathrm{FEV}_{1}(\mathrm{SD}), \%$ of predicted & $56.9(16.6)$ & $57.0(16.7)$ & $56.9(16.6)$ \\
\hline \multicolumn{4}{|l|}{ Exacerbations in the previous year, $n(\%)$} \\
\hline None & $190(71)$ & $76(69)$ & $114(73)$ \\
\hline$\geq 1$ & $76(29)$ & $34(31)$ & $42(27)$ \\
\hline Mean CRQ dyspnea scores (SD) & $4.9(1.5)$ & $4.6(1.5)$ & $5.1(1.5)$ \\
\hline Mean feeling thermometer scores (SD) & $69.9(15.3)$ & $67.4(15.7)$ & $71.6(14.8)$ \\
\hline \multicolumn{4}{|l|}{ Mean HADS scores (SD) } \\
\hline Depression & $4.3(3.6)$ & $4.8(3.9)$ & $3.9(3.4)$ \\
\hline Anxiety & $4.8(4.2)$ & $5.6(4.6)$ & $4.2(3.8)$ \\
\hline \multicolumn{4}{|l|}{ Medication use, $n(\%)$} \\
\hline Depression & $30(11)$ & $17(15)$ & $13(8)$ \\
\hline Anxiety & $28(11)$ & $21(19)$ & $7(4)$ \\
\hline Inhaled corticosteroids & $156(59)$ & $65(59)$ & $91(58)$ \\
\hline Long-acting $\beta$-agonists & $152(57)$ & $59(54)$ & $93(60)$ \\
\hline
\end{tabular}

BMI, body mass index; CRQ, Chronic Respiratory Disease Questionnaire; $\mathrm{FEV}_{1}$, forced expiratory volume in $1 \mathrm{~s}$; HADS, Hospital Anxiety and Depression Scale; SD, standard deviation.

those who did not, and we adjusted for sex, age (continuous), living status (alone or not), and lung function (forced expiratory volume in $1 \mathrm{~s}$ ). For the adjusted analysis of depression, we excluded patients who had clinically important depression symptoms (HADS score $\geq 11$ ) at baseline, and, similarly, for the adjusted analysis of anxiety, we excluded patients who had clinically important anxiety symptoms (HADS score $\geq 11$ ) at baseline. For other outcomes, such patients were not excluded; instead, we adjusted for baseline clinically important depression/anxiety status (yes vs. no) in the regression models. Next, interaction terms of stressful life events with the other variables were added to the models to assess if any subgroups of patients were more vulnerable to the impact of stressful life events. All analyses were done using Stata, version 13 (StataCorp LP, College Station, TX, USA).

\section{Results}

The ICE COLD ERIC study recruited 409 patients, and our sample included 266 patients who remained in the study (not dead or lost to follow-up) at the 3.5-year visit and completed the assessment of stressful life events. 
Table 2. Categories of reported stressful life events

\begin{tabular}{llr}
\hline & $\begin{array}{l}\text { Events reported } \\
N / 145, \%\end{array}$ & $\begin{array}{l}\text { Events rated as “extremely } \\
\text { stressful," } n / N, \%\end{array}$ \\
\hline Death of relatives/important persons & $45(31)$ & $11(24)$ \\
Patient's own disease & $30(21)$ & $5(17)$ \\
Disease of relatives/important persons & $28(19)$ & $11(39)$ \\
Relational problems with relatives/important persons & $15(10)$ & $3(20)$ \\
Financial worries & $10(7)$ & $4(40)$ \\
Unemployment/retirement & $5(3)$ & $1(20)$ \\
Divorce/separation/loss of friendship & $5(3)$ & $2(40)$ \\
Loss of pets & $3(2)$ & $2(67)$ \\
Other & $4(3)$ & $0(0)$ \\
\hline
\end{tabular}

a Total number of events $=145$.

The study flow chart can be found in the online supplementary materials (for all online suppl. material, see www.karger.com/doi/10.1159/000481714), and patients' characteristics at baseline are shown in Table 1. Fortyone percent (110/266) of the patients reported having experienced any stressful life events. Those who had experienced stressful life events were younger and less likely to be retired than those who had not experienced stressful life events. Another noticeable difference was that those who had experienced stressful life events ascribed lower scores to their dyspnea-related quality of life and health status and reported more symptoms of depression and anxiety at study baseline. More of these patients were also taking depression and anxiety medications. The study sample included slightly more males (154/266, $58 \%)$.

One hundred and fifty-six (59\%) patients reported not having experienced any stressful life events, 82 (31\%) reported $1,21(8 \%)$ reported 2 , and $7(3 \%)$ reported 3 events. The most commonly reported event by patients was "death of relatives/important persons," followed by "patient's own disease" and "disease of relatives/important persons" (see Table 2, also presenting the number of patients who rated each event as "extremely stressful").

The median time at which the patients reported having experienced the first event was between visit 3 (1.5-year follow-up visit) and 4 (2-year follow-up visit); the 25th percentile was between visit 1 (0.5-year follow-up visit) and 2 (1-year follow-up visit); the 75th percentile was between visit 5 (2.5-year follow-up visit) and 6 (3-year follow-up visit). In 18 patients, the dates of all their reported stressful life events were before study baseline. These patients were counted as having "no stressful life events" in the analysis assessing the impact of stressful life events on patient outcomes since all outcome data were collected from baseline to the 3.5-year visit.

Table 3 summarizes the results that assessed the impact of stressful life events on health outcomes/behaviors. The within-patient comparison for patients who had stressful life events suggests that, over time, these patients' symptoms of depression increased and their dyspnea-related quality of life and health status decreased. The numbers of cigarettes smoked per day and their levels of physical activity decreased. In the multivariable linear regression analysis of the before-after change, we found that comparing those who reported any stressful life events with those who reported none, those reporting stressful life events had a greater increase in depression symptoms (coefficient $0.9 ; 95 \%$ confidence interval [CI] 0.3 to 1.4 ) and a greater increase in anxiety symptoms (coefficient 0.8 ; $95 \%$ CI 0.3 to 1.3 ).

To aid in interpretation, we defined "responders with depression/anxiety symptoms" to be patients who had a 1.5-unit increase on the HADS depression/anxiety subscale (minimal important difference [20]), comparing the "after period" with the "before period." We found that among those who reported stressful life events, 38\% (35/91) were responders with depression symptoms, while 25\% (44/174) among those who reported no stressful life events were responders with depression symptoms $\left(\chi^{2}\right.$ statistic $=4.96, p$ value $\left.=0.026\right)$. For anxiety symptoms, 30\% (27/91) among those who reported stressful life events were responders with anxiety symptoms, and $13 \%(22 / 174)$ among those who reported no stressful life events were responders with anxiety symptoms $\left(\chi^{2}\right.$ statistic $=11.49, p$ value $=0.001)$. Stressful life events were associated with more responders. 
Table 3. Summary of results assessing the impact of stressful life events on health outcomes and health behaviors

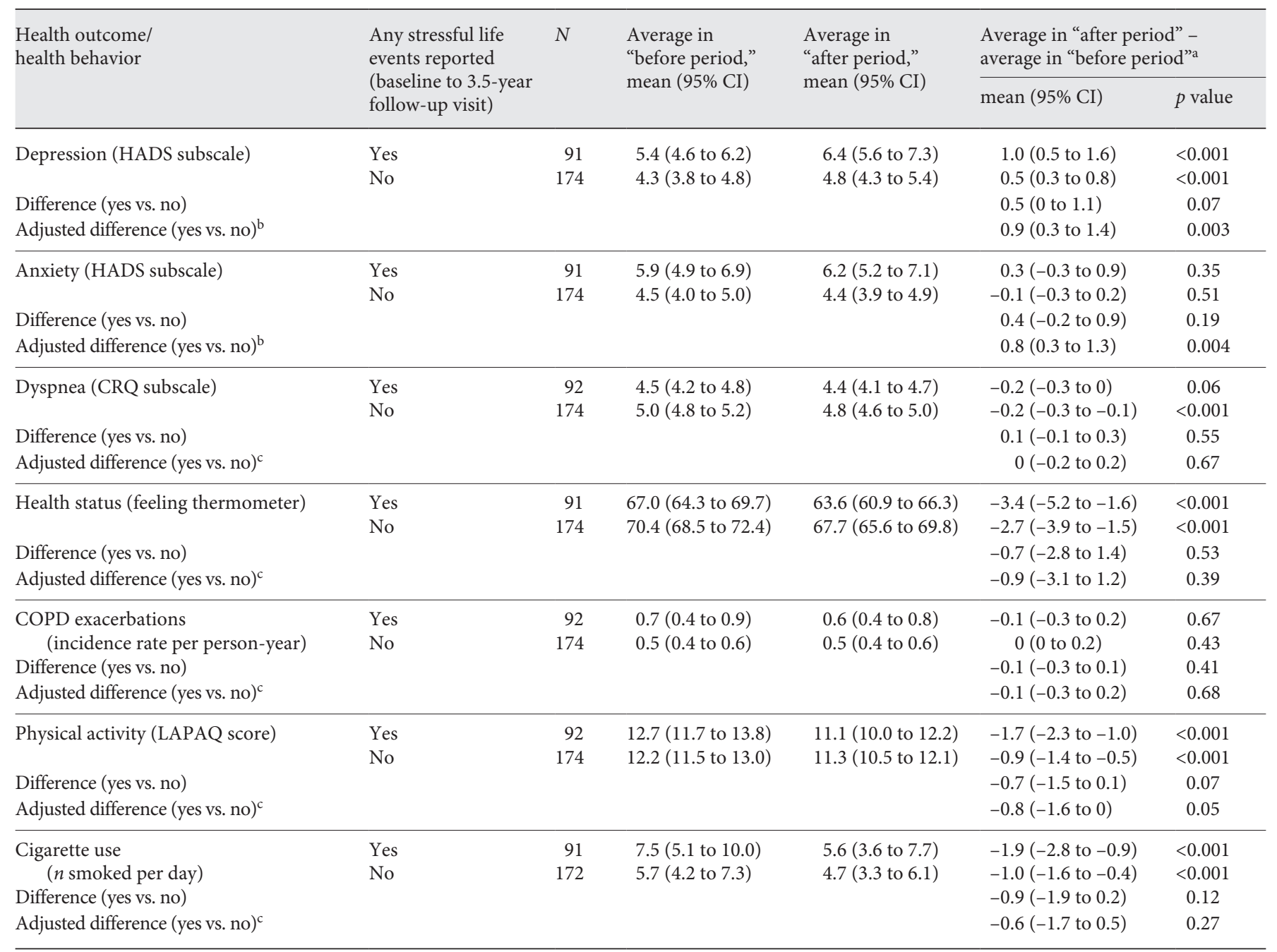

CI, confidence interval; COPD, chronic obstructive pulmonary disease; CRQ, Chronic Respiratory Disease Questionnaire; HADS, Hospital Anxiety and Depression Scale; LAPAQ, Longitudinal Aging Study Amsterdam Physical Activity Questionnaire. ${ }^{\text {a }}$ For patients who had any stressful life event, "before period" is the time period before the first event and "after period" is the time period after the first event. For patients who had no stressful life event, "before period" is the time period before visit 4 and "after period" is the time period from visit 4 onwards. ${ }^{\text {b }}$ We excluded patients who had baseline clinically important depression (for depression outcome) or anxiety (for anxiety outcome) and adjusted for age, sex, living status, and lung function in the regression analysis. ${ }^{\mathrm{c}}$ We adjusted for age, sex, living status, lung function, and depression/anxiety status at baseline in the regression analysis.

Regarding health behaviors, patients who reported any stressful life events had a larger decrease in physical activity (coefficient $-0.8 ; 95 \%$ CI -1.6 to 0 ) than those who reported none, while no difference was found for cigarette use.

In the online supplementary materials, we reported the results of the multivariable linear regression analyses where we added interaction terms of stressful life events with each of the following variables: sex, age, living status, lung function, and baseline depression/anxiety status.
There was no indication of any interactions for any of the outcomes/behaviors.

\section{Discussion}

In our study, we found that almost half of the COPD patients reported to have experienced events that were so stressful that they seriously impacted their lives. Our analyses suggest that experiencing stressful life events is 
associated with increased future symptoms of depression and anxiety, while no effect of stressful life events on other physical outcomes, including dyspnea, health status, and COPD exacerbations, was identified. We also found that stressful life events may be associated with a future decrease in physical activity.

The 3 most commonly reported stressful life events by our study participants were "death of relatives/important persons" (31\%), "patient's own disease" (21\%), and "disease of relatives/important persons" (19\%), accounting for more than $70 \%$ of all events. The frequency of diseaserelated events agrees with previous studies on stressful life events done in elderly populations, where health-related events were found to be the most frequent type of stress in older adults $[21,22]$. Different events were perceived differently by our study participants. For example, among the 3 most frequently reported types of stressful life events, the proportion of patients who rated the event as "extremely stressful" was higher for "disease of relatives/ important persons" than for "death of relatives/important persons" or "patient's own disease." The reason why "disease of relatives/important persons" was perceived as a more stressful event may be that the informal care given to those getting sick can be a substantial burden to most caregivers. It would also be interesting to learn whether these health-related events are perceived differently from those events that are not health related (e.g., financial worries, unemployment, and divorce). However, due to the small numbers of these events reported in our study, we cannot make such comparisons.

Patients who reported stressful life events differed from those who did not report any stressful life events at baseline regarding the levels of symptoms of depression and anxiety, dyspnea-related quality of life, and health status. Given that our instrument to assess stressful life events relied on patients' recall of experience in the past years, it is likely that patients who were more depressed/anxious, had more severe dyspnea, and rated their health status as lower at baseline had a higher chance of remembering or reporting stressful life events. Also, such patients might be more prone to perceive an event as being "stressful," or they might be less resilient to the same event than patients who were less depressed/anxious, had less severe dyspnea, and rated their health status as higher at baseline $[6,23]$. Furthermore, there can be some strong confounding factors (e.g., individual personality traits) that are linked to patients' symptoms, such as depression/anxiety, and at the same time affect their risk of experiencing stressful life events $[6,23]$. Faced with such an imbalance in the baseline characteristics, when doing the analysis to assess the impact of stressful life events on patient outcomes/behaviors, we took advantage of our longitudinal study design and within-patient comparison.

According to the 2 main pathways proposed to link stressful life events with health problems, psychological stress may lead to physiological dysregulation or to worse health behaviors, which in turn increase the risk for adverse outcomes [9]. In our study, we computed the within-patient before-after change of health outcomes and behaviors. We tried to examine whether this within-patient change was more pronounced in patients who reported having experienced any stressful life events. After accounting for confounders, we observed that stressful life events were associated with more symptoms of depression and anxiety. There seemed to be an association of stressful life events with less physical activity, but no association with cigarette use was found. On the other hand, our analysis of stressful life events and physical outcomes did not suggest an association with COPD exacerbations and dyspnea-related quality of life, and the finding of a change in health status had a high variance. Because our study sample size was relatively small and our time frame of follow-up after the event was relatively short, more replications of these analyses in other COPD cohorts are needed in the future to examine the impact of stressful life events on COPD prognosis.

Our study has several limitations. First, the stressful life events were retrospectively assessed by asking the participants to recall the experience and dates of stressful life events for the past 3 years. This perhaps posed some challenge to the participants of this age group. Our study design could have been improved if a shorter time frame had been chosen (e.g., 1 year) for patients to recall their experience of stressful life events and if we had assessed this factor repeatedly. However, most stressful life events are significant events in patients' life, so they should have been easier for patients to remember. Second, symptoms of depression and anxiety were assessed by self-reported questionnaires. We need to bear in mind that these are not a formal diagnosis of depression and anxiety, which will require a clinical interviewer and information from medical records or other informants. Third, because assessments of stressful life events and most outcomes were completed by patients alone, there is a risk that the differences observed in the current study were confounded in part by this dependency upon self-reporting.

Despite these limitations, we believe that the present study still makes a valuable contribution to the current knowledge on stressful life events in COPD patients and on the influence of stressful life events on COPD out- 
comes. With data from a longitudinal follow-up study, we were able to demonstrate that experience of stressful life events may affect mental health, including more symptoms of depression and anxiety in patients with COPD, while we did not obtain similar findings for other physical outcomes. Stressful life events may also lead to a decrease in physical activity. The importance of mental health status for patients with COPD has been well recognized by the clinical community over the past few years. Finding effective ways to cope with the stress encountered by COPD patients can be one of the future steps to reduce their risk of developing depression and anxiety.

\section{Acknowledgements}

We thank Ursula Schafroth (Horten Centre for patient-oriented research, University of Zurich, Switzerland) and Alice Karsten (Academic Medical Centre, Department of General Practice, University of Amsterdam, The Netherlands) and the participating general practitioners and patients with COPD in Switzerland and the Netherlands (Stichting Gezondheidscentra Amsterdam Zuidoost and Zorggroep Almere) who made this study possible by their enthusiastic participation.

\section{Financial Disclosure and Conflicts of Interest}

The authors declare that they have no conflicts of interest. The sponsors had no role in the design of the study, the collection and analysis of the data, or the preparation of the manuscript.

\section{Funding Sources}

Funding was provided by the Swiss National Science Foundation (grant number 3233B0/115216/1), the Dutch Asthma Foundation (grant number 3.4.07.045), and the Zurich Lung League (unrestricted grant).

\section{Author Contributions}

M.A.P. had full access to all data in the study and takes responsibility for the integrity of the data and the accuracy of the data analysis. T.Y., M.A.P., and A.F. contributed to the conception and design of the study. T.Y., G.R., M.A.P., and A.F. contributed to data analysis and interpretation of data. T.Y. drafted the manuscript. T.Y., G.R., M.A.P., and A.F. critically revised the manuscript and approved the final version.

\section{References}

1 Cohen S, Janicki-Deverts D, Miller GE: Psychological stress and disease. JAMA 2007;298: 1685-1687.

2 Tosevski DL, Milovancevic MP: Stressful life events and physical health. Curr Opin Psychiatry 2006;19:184-189.

3 Holmes TH, Rahe RH: The Social Readjustment Rating Scale. J Psychosom Res 1967;11: 213-218.

4 Blazer D, Hughes D, George LK: Stressful life events and the onset of a generalized anxiety syndrome. Am J Psychiatry 1987; 144:11781183.

5 Duijts SF, Zeegers MP, Borne BV: The association between stressful life events and breast cancer risk: a meta-analysis. Int J Cancer 2003;107:1023-1029.

6 Kessler RC: The effects of stressful life events on depression. Annu Rev Psychol 1997;48: 191-214.

7 Krantz DS, McCeney MK: Effects of psychological and social factors on organic disease: a critical assessment of research on coronary heart disease. Annu Rev Psychol 2002;53: 341-369.

8 Porcelli B, et al: Association between stressful life events and autoimmune diseases: a systematic review and meta-analysis of retrospective case-control studies. Autoimmun Rev 2016;15:325-334.
9 Sarafino EP, Smith TW: Health Psychology: Biopsychosocial Interactions, ed 8. John Wiley \& Sons, 2014.

10 Atlantis E, et al: Bidirectional associations between clinically relevant depression or anxiety and COPD: a systematic review and metaanalysis. Chest 2013;144:766-777.

11 Laurin C, et al: Impact of anxiety and depression on chronic obstructive pulmonary disease exacerbation risk. Am J Respir Crit Care Med 2012;185:918-923.

$12 \mathrm{Lu} \mathrm{Y}$, et al: Life event stress and chronic obstructive pulmonary disease (COPD): associations with mental well-being and quality of life in a population-based study. BMJ Open $2012 ; 2$.

13 Siebeling L, et al: ICE COLD ERIC - international collaborative effort on chronic obstructive lung disease: exacerbation risk index cohorts - study protocol for an international COPD cohort study. BMC Pulm Med 2009;9: 15.

14 Siebeling L, et al: Characteristics of Dutch and Swiss primary care COPD patients - baseline data of the ICE COLD ERIC study. Clin Epidemiol 2011;3:273-283.

15 Zigmond AS, Snaith RP: The hospital anxiety and depression scale. Acta Psychiatr Scand $1983 ; 67: 361-370$
16 Guyatt GH, et al: A measure of quality of life for clinical trials in chronic lung disease. Tho$\operatorname{rax} 1987 ; 42: 773-778$.

17 EuroQol Group: EuroQol - a new facility for the measurement of health-related quality of life. Health Policy 1990;16:199-208.

18 Puhan MA, et al: Relative responsiveness of the Chronic Respiratory Questionnaire, St. Georges Respiratory Questionnaire and four other health-related quality of life instruments for patients with chronic lung disease. Respir Med 2007;101:308-316.

$19 \mathrm{Yu} \mathrm{T}$, et al: Determinants of physical activity in patients with chronic obstructive pulmonary disease: a 5-year prospective follow-up study. Respiration 2016;92:72-79.

20 Puhan MA, et al: The minimal important difference of the Hospital Anxiety and Depression Scale in patients with chronic obstructive pulmonary disease. Health Qual Life Outcomes 2008;6:46.

21 Glass TA, Kasl SV, Berkman LF: Stressful life events and depressive symptoms among the elderly. Evidence from a prospective community study. J Aging Health 1997;9:70-89.

22 Hardy SE, Concato J, Gill TM: Stressful life events among community-living older persons. J Gen Intern Med 2002;17:832-838.

23 Kendler KS, Karkowski LM, Prescott CA: Causal relationship between stressful life events and the onset of major depression. Am J Psychiatry 1999;156:837-841. 